\title{
IFN- alpha blocks IL-17 production by peripheral blood mononuclear cells in patients with chronic active hepatitis B Infection
}

\author{
Fang Cui ${ }^{1 *}$, Jiangping Meng ${ }^{2}$, Peng Luo ${ }^{1}$ and Pu Chen ${ }^{1}$
}

\begin{abstract}
Background: IFN-a has been used to treat patients with chronic active hepatitis B (CAHB). Recent studies have implicated the IL-23/Th-17 pathway in the pathogenesis of CAHB. In this study, we investigated whether IFN-a could affect this pathway.

Methods: Peripheral blood mononuclear cells (PBMCs) obtained from patients with active CAHB $(n=61)$ and controls ( $n=32$ ) were cultured with or without IFN- , and the levels of IL-17 and IL-10 in the supernatants were determined by ELISA, while the frequency of IL-17-expressing cells was measured by FACS. Similar experiments were also conducted with isolated CD4+ T cells from controls. Furthermore, an experiment using an anti-IL-10 antibody was performed to examine the underlying mechanisms of action of IFN-a.
\end{abstract}

Results: Both the levels of IL-17 and the frequency of IL-17-expressing cells were significantly higher in the PBMCs from CAHB patients than in the controls. IFN-a significantly decreased IL-17 production and the frequency of IL-17expressing cells in PBMCs from both patients and controls. On the other hand, IFN-a increased IL-10 production by PBMCs from patients and controls. Anti-IL-10 antibody was able to neutralize the inhibitory effect of IFN-a on IL-17 production by PBMCs.

Conclusions: In vitro experiments showed that IFN-a could inhibit IL-17 expression and increase IL-10 production by PBMCs and CD4+ T cells. The inhibitory role of IFN-a on IL-17 production was partly mediated by IL-10.

Keywords: Chronic hepatitis B infection, IFN-a, IL-17, IL-10

\section{Background}

Hepatitis B virus (HBV) infection, the major cause of chronic liver injury and inflammation, can lead to liver fibrosis, cirrhosis and hepatocellular carcinoma [1,2]. IFN- $\alpha$ has been used for the treatment of HBV infectionassociated complications with promising results. The mechanisms whereby IFN- $\alpha$ exerts its immunomodulatory effects in HBV-infected patients are, however, not completely understood.

It was suggested that the IL-23/Th-17 pathway is implicated in the pathogenesis of the immune response to promote liver damage $[3,4]$, and that the effective treatment of CAHB patients with IFN- $\alpha$ might be mediated

\footnotetext{
* Correspondence: cuihpp@126.com

'Department of Laboratory Medicine, The First Affiliated Hospital of Chongqing Medical University, No. 1 Youyi Road, Chongqing, Yuzhong District, China

Full list of author information is available at the end of the article
}

via this pathway. Recent studies have shown that Type I IFNs, including IFN- $\alpha$, could inhibit IL-17 production by peripheral blood mononuclear cells (PBMCs) [5], and Type I IFNs were able to stimulate IL-10 production by activated $\mathrm{CD}^{+}{ }^{+} \mathrm{T}$ cells [6]. IL-10 is a cytokine of T-regulatory Type 1 cells that can inhibit the production of IFN- $\gamma$ [7] and antigen presentation [8]. It has also been reported that IL-10 can restrain the pathogenic role of Th17 cells [9]. However, it is not yet known whether IFN- $\alpha$ can inhibit IL-17 in CAHB, and, if so, whether it inhibits IL-17 production directly or indirectly (for instance, through the regulation of IL-10 expression). This study was designed to investigate whether IFN- $\alpha$ could inhibit IL-17 production by PBMCs in CAHB patients and to elucidate the mechanism that is involved in this process. Our results showed that IFN- $\alpha$ could significantly inhibit IL-17 production in association with 
increased IL-10 production by PBMCs from both CAHB patients and controls. Furthermore, similar findings were confirmed using isolated $\mathrm{CD}^{+} \mathrm{T}$ cells from controls. Interestingly, we found that the inhibitory role of IFN $-\alpha$ on IL-17 production was partially mediated by IL- 10 .

\section{Methods}

Study population

Sixty-one inpatients with CAHB were enrolled in the study (38 men and 23 women with an average age of 38.7 years). The diagnostic and treatment criteria were based on the clinical practice guidelines of the "European Association for the Study of the Liver" [10], and the Asian-Pacific consensus statement on the management of chronic hepatitisB: a 2012 update [11]. For all patients, there was no evidence of metastatic or autoimmune liver disease. The inclusion criteria were: serum HBsAg positive for at least 6 months, alanine aminotransferase (ALT) $>2$ times of the normal level $(<72 \mathrm{IU})$, and Log HBV DNA $>5$, but no evidence for HCC, or concomitant of HCV, HDV, HIV infection and autoimmune liver disease. The excluded criteria were: exposure to nucleos $(t)$ ide analog(s), acute hepatitis A, B, HCV, HDV, or HIV co-infection, and drug-induced acute hepatitis, existence of renal failure, hepatic decompensation or psychiatric disorders, and central nervous system diseases, such as epilepsy, bone marrow or organ transplants, or immunosuppressive, nephrotoxic, or hepatotoxic medications received within 2 months of enrollment. Thirty-two healthy individuals (17 men and 15 women, with an average age of 33.7 years) acted as controls. Patients and controls were included in this study between October 2012 and November 2013. They were mainly from different regions of Southwest China. Written informed consent according to the Declaration of Helsinki was obtained from each individual, and the above protocol was approved by the Clinical Research Ethics Committee of the first affiliated hospital of Chongqing Medical University. There were no significant differences in age and gender proportion between patients and healthy volunteers (Table 1).

\section{Cell isolation and culture}

PBMCs were isolated from heparinized blood samples by Ficoll-Hypaque density-gradient centrifugation. Peripheral CD4+ T cells of controls were isolated from PBMCs by human CD4 microbeads (Miltenyi Biotec, Palo Alto, CA, USA) according to the manufacturer's instructions. The purity of CD4+ T cells was detected using FACS Canton II and FACS Diva software (BD Company, Franklin Lakes, NJ, USA) after staining with anti-human CD4 antibodies (BD Company, USA). The result showed that the purity was $>92 \%$ in each experiment. PBMCs were cultured for $72 \mathrm{~h}$ with or without rhIFN- $\alpha$ 2a (PBL Biomedical Lab, Piscataway, NJ, USA), anti-IL-10 (R\&D Systems) at a
Table 1 Demographic and clinical characteristics of the subjects enrolled in the study

\begin{tabular}{lll}
\hline & Healthy controls $(\mathbf{n}=\mathbf{3 2})$ & CAHB $(\mathbf{n}=\mathbf{6 1})$ \\
\hline Age, years & & \\
Mean & 32.9 & 38.7 \\
SD & 14.2 & 13.8 \\
Male/female & $17 / 15$ & $38 / 23$ \\
HBsAg & - & + \\
Log HBV DNA & & \\
Mean & - & 6.34 \\
SD & $/$ & 1.36 \\
Serum ALT (IU/liter) & & \\
Mean & 15 & 179.4 \\
SD & 5.6 & 33.2 \\
\hline
\end{tabular}

concentration of $1 \times 10^{6}$ cells $/ \mathrm{ml}$ in combination with antiCD3 $(5 \mathrm{mg} / \mathrm{ml})$ and anti-CD28 anti- bodies $(1 \mathrm{mg} / \mathrm{ml})$ (eBioscience, San Diego, CA, USA). CD4+ T cells $\left(1 \times 10^{6}\right.$ cells $/ \mathrm{ml}$ ) were cultured for $72 \mathrm{~h}$ with or without rhIFN- $\alpha$ 2a $(1 \mathrm{mg} / \mathrm{ml})$ in combination with anti-CD3 $(5 \mathrm{mg} / \mathrm{ml})$ and anti-CD28 antibodies $(1 \mathrm{mg} / \mathrm{ml}$ ) (Miltenyi Biotec, Germany). Supernatants from these cell cultures were used for detecting the concentration of IL-17 and IL-10.

\section{Intracellular cytokine staining}

As a longer incubation period was needed to study the influence of IFN- $\alpha$ on the IL-17 production, we first compared the result of 20-h and 6-h incubation with phorbol myristate acetate (PMA) and ionomycin in five samples. Our result showed that there was no significant difference concerning both IL-17 expression and the frequency of live PBMCs between 20-h and 6-h incubation. Therefore, a 20-h incubation was used in the following experiments. Briefly, PBMCs from patients and controls were cultured with or without rhIFN- $\alpha$ 2a (PBL Biomedical Lab, Piscataway, NJ, USA), at a concentration of $2 \times 10^{6}$ cells $/ \mathrm{ml}$ in combination with $20 \mathrm{ng} / \mathrm{ml}$ PMA and $1 \mu \mathrm{g} / \mathrm{ml}$ ionomycin (Sigma, Saint Louis, MO, USA), for $20 \mathrm{~h}$ and then blocked with $10 \mu \mathrm{g} / \mathrm{ml}$ brefeldin A (Sigma, St Louis, MO, USA) for $4 \mathrm{~h}$. The cultured cells were stained with anti-CD3, anti-CD4 antibodies (BD Company, USA), washed, fixed, permeabilized, and subsequently stained with anti-IL-17A (BD Company, USA) or appropriate isotypes (eBioscience, San Diego, CA). FACS analysis was performed using FACS Canton II and FACS Diva software (BD Company, USA) to determine the in vitro effect of IFN- $\alpha$ on the expression of IL-17.

\section{ELISA}

Peripheral blood samples of patients and normal controls were collected and placed in tubes free of pyrogen and endotoxin. After centrifugation at a speed of $1000 \times g$ for 15 min, serum samples were harvested and 
stored at $-70^{\circ} \mathrm{C}$ until use. The concentration of IL-17 and IL-10 in the supernatants of cultured PBMCs and $\mathrm{CD}^{+} \mathrm{T}$ cells was measured using the human Duoset ELISA development kit (R\&D systems), which has a detection limit of 15 and $31 \mathrm{pg} / \mathrm{ml}$, respectively.

\section{Statistical analysis}

Paired-sample $t$-test was applied for within group comparison and one-way ANOVA was applied for between group comparison using SPSS10.0 software (SPSS Inc., Chicago, IL, USA). Data are expressed as mean \pm SEM. $\mathrm{p}<0.05$ was considered as statistically significant.

\section{Results}

Concentration of IL-17 in the supernatants is higher in the cultured PBMCs from CAHB patients than in the controls and in both addition of rhIFN-a decreases IL-17 production.

ELISA was performed to determine IL-17 in the supernatants of stimulated PBMCs from patients and controls. Stimulation of PBMCs with anti-CD3 and anti-CD28 antibodies resulted in significant production of IL-17 ( $p<0.001)$. IL-17 levels in the cell culture supernatants of PBMCs from CAHB patients were significantly higher than those obtained from controls $(\mathrm{p}<0.037)$. Addition of rhIFN- $\alpha 2 \mathrm{a}$ to this cell culture model revealed a significantly decreased IL-17 production both in CAHB patients and controls (Figure 1).

The frequency of IL-17-expressing CD4+ T cells is significantly increased in CAHB patients and addition of rhIFN- $a$ 2a decreases the frequency of IL-17 expressing cells from both patients and controls

We further analyzed the frequency of IL-17- expressing T cells in CAHB patients $(n=61)$ and controls $(n=32)$ using FACS analysis. We found that both $\mathrm{CD}^{+}$and
CD4-T cells expressed IL-17, but since the frequencies of IL-17-expressing CD4-T cells were extremely low in CAHB patients, we only investigated the $\mathrm{CD} 4^{+} \mathrm{T}$ cells. We found that frequency of IL-17-expressing CD4 ${ }^{+} \mathrm{T}$ cells was significantly increased in CAHB patients compared to healthy controls $(\mathrm{p}<0.001)$. Addition of rhIFN- $\alpha$ 2a could decrease the frequencies of IL-17-expressing $\mathrm{T}$ cells in CAHB patients and in controls $(\mathrm{p}<0.001)$ (Figure 2).

\section{rhIFN-a $2 a$ increases IL-10 production by PBMCs from CAHB patients and controls}

ELISA was also performed to determine the levels of IL10 in the supernatants of stimulated PBMCs from patients and controls. IL-10 levels in the cell culture supernatants of PBMCs from CAHB patients were significantly higher than those obtained from controls $(p<0.001)$. Addition of rhIFN- $\alpha 2 \mathrm{a}$ to this cell culture model revealed a significant increase of IL-10 production $(\mathrm{p}<0.001)$ (Figure 3$)$.

rhIFN-a 2a inhibits IL-17 production, but increases IL-10 production by isolated $\mathrm{CD}^{+} \mathrm{T}$ cells from healthy controls Experiments using isolated $\mathrm{CD}^{+}{ }^{+} \mathrm{T}$ cells from healthy controls showed that rhIFN- $\alpha$ 2a also significantly inhibited IL-17 production $(\mathrm{p}<0.001)$ and promoted IL-10 production $(\mathrm{p}<0.001)$ (Figure 4). IL-17 and IL-10 levels were much higher in experiments using isolated $\mathrm{CD}^{+}{ }^{+} \mathrm{T}$ cells from healthy individuals as compared with those in PBMCs.

\section{Anti-IL-10 antibody partially neutralizes the inhibitory effect of rhIFN-a 2a on IL-17 production by PBMCs from CAHB patients}

A significant decrease of IL17 production by PBMCs $(\mathrm{p}<0.001)$ (Figure 1) was observed in the presence of

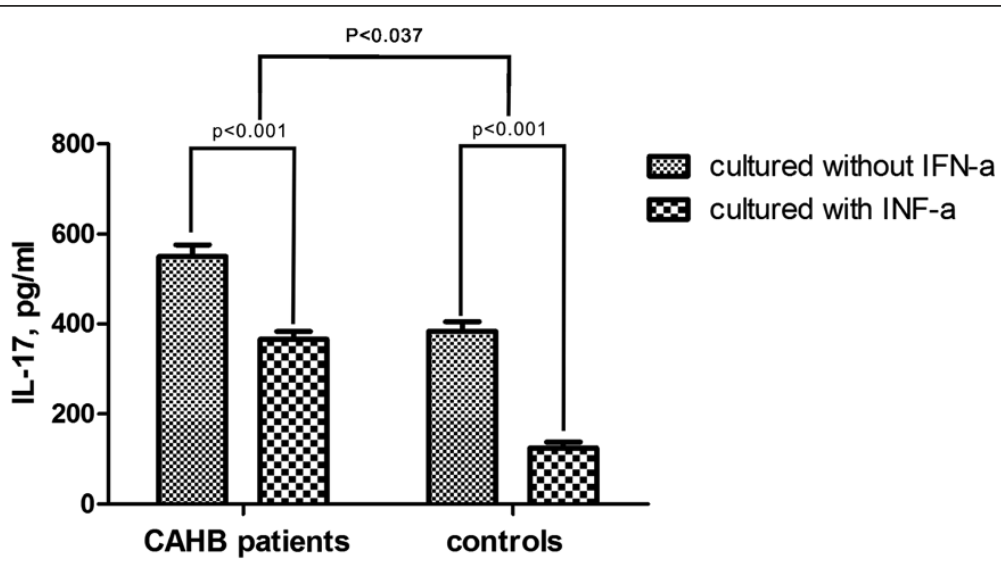

Figure 1 The inhibitory effect of rhIFN- $a$ 2a on IL-17 production from PBMCs from CAHB patients $(n=61)$ and healthy controls $(n=32)$. PBMCs from CAHB patients $(n=61)$ and healthy controls $(n=32)$ were cultured with or without rhIFN-a 2a at a concentration of $1 \times 10^{6}$ cells $/ \mathrm{ml}$ in combination with anti-CD3 $(5 \mathrm{mg} / \mathrm{ml})$ and anti-CD28 anti- bodies $(1 \mathrm{mg} / \mathrm{ml})$ for $72 \mathrm{~h}$. IL-17 production in the supernatant of cultured PBMCs was measured by ELISA. Columns represent meanvalues (mean + SEM). 

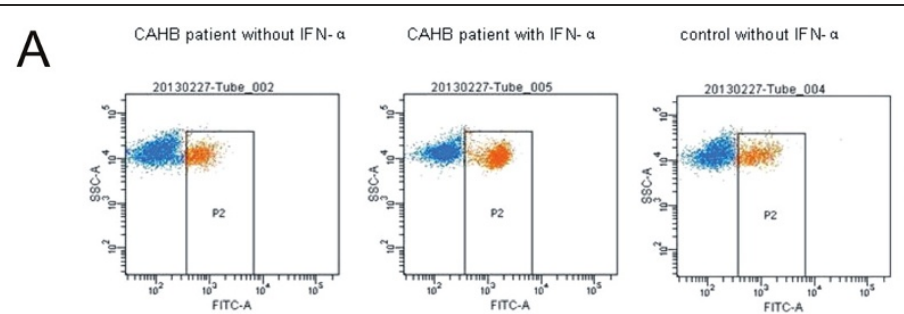

control with IFN-a
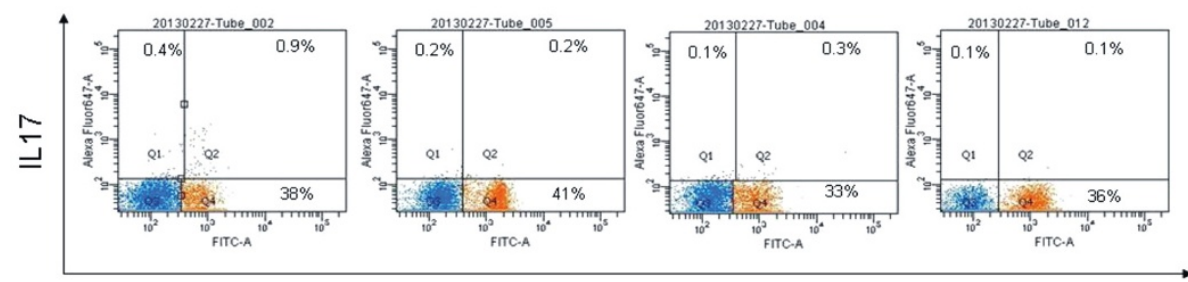

CD4

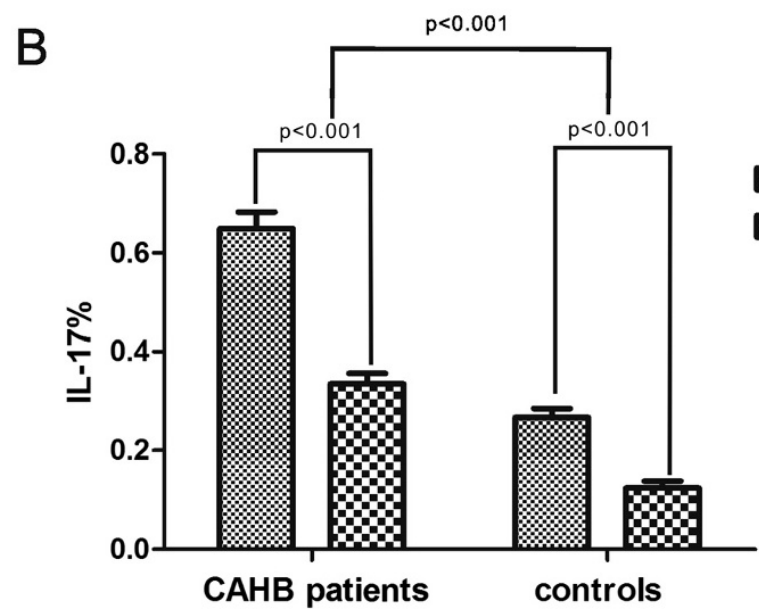

Figure 2 Detection the Frequencies of IL-17 expressing CD4+ T cells in PBMCs from CAHB patients $(n=61)$ and healthy controls $(n=32)$ cultured with or without rhIFN- $\alpha$ 2a by intracellular cytokine staining assay and FACS analysis. (A) Data from a representative patient. Numbers indicate percentages of positive cells in that quadrant. (B) Quantitative analysis of IL-17 -expressing CD4+ T cells. Columns represent mean values (mean + SEM).

IFN- $\alpha$, which was associated with an elevated IL-10 level $(\mathrm{p}<0.001)$ (Figure 3). To investigate the role of IL-10, we incubated PBMCs in the presence of IFN- $\alpha$ and an antibody against IL-10. In the presence of this antibody against IL-10, the inhibitory effect of rhIFN- $\alpha$ 2a on IL-17 production was partially blocked ( $\mathrm{p}=0.022)$ (Figure 5).

\section{Discussion}

In the present study, IL-17 levels in the cell culture supernatants of PBMCs from CAHB patients were significantly higher than those obtained from controls. Earlier studies showed that the level of IL-17 was elevated in CHB patients with high ALT level and suggested that IL-17 is associated with the active intrahepatic inflammation in $\mathrm{CHB}$ patients.

In recent studies of patients with autoimmune hepatitis [12] and hepatitis B [3], the degree of hepatic inflammation was directly correlated with Th17 cell infiltration. In our study, the frequency of peripheral Th17 cells in CAHB patients was found to be significantly higher than that of normal controls. The frequency of Th17 cells in the PBMC population was examined after stimulation with PMA and ionomycin, which are pharmacological $\mathrm{T}$ cell-activating agents that mimic signals generated by the $\mathrm{T}$ cell receptor (TCR) complex. However, this combinatory stimulation did not replicate the physiological conditions by which $\mathrm{T}$ cells are stimulated in vivo.

Our results are also consistent with those reported earlier in CAHB patients. Th17 cells were found to be highly enriched in the peripheral blood and liver, and exhibited a significant potential to exacerbate liver damage during HBV infection [3]. The frequency of peripheral Th17 cells also correlated with ALT, suggesting a potential role for Th17 cells in the immune activation of chronic HBV infection [13]. Recently, it was reported that the 


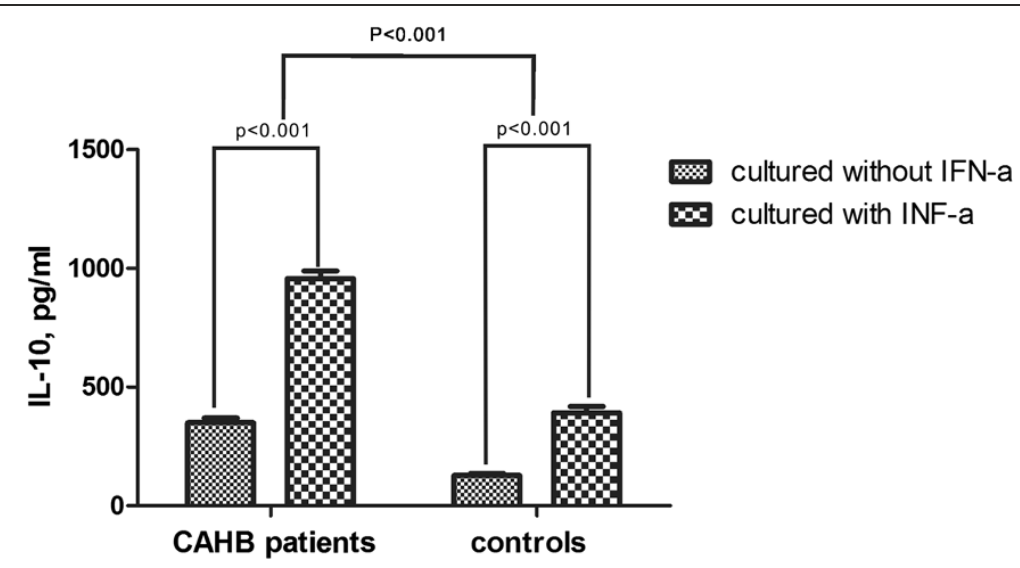

Figure 3 rhIFN-a 2a increase the production of IL-10 from PBMCs from CAHB patients $(n=61)$ and healthy controls $(n=32)$. PBMCs from CAHB patients $(n=61)$ and healthy controls $(n=32)$ were cultured with or without rhIFN- $a$ 2a at a concentration of $1 \times 10^{6}$ cells $/ \mathrm{ml}$ in combination with anti-CD3 $(5 \mathrm{mg} / \mathrm{ml})$ and anti-CD28 anti- bodies $(1 \mathrm{mg} / \mathrm{ml})$ for $72 \mathrm{~h}$. IL-10 production in the supernatant of cultured PBMCs was measured by ELISA. Columns represent meanvalues (mean + SEM).

frequency of circulating Th17 cells in HBV-infected patients positively correlated with the severity of the disease and the extent of liver injury. Therefore, it has been hypothesized that Th17 cells might contribute to HBV disease progression and development of liver injury $[4,14]$.

IL-10 is an anti-inflammatory cytokine involved in the down-regulation of the immune response. Research has indicated that induction of IL-10 can limit proinflammatory Th17 responses in HBV infected patients [15]. The strong correlation between HBV load and the regulatory T cell (Treg)/Th17 ratio in CAHB indicates that the imbalance of these two cell types might contribute to HBV persistence. Th17-mediated inflammatory diseases [16] strongly support the notion that Th17 cells play a proinflammatory role in the pathogenesis of hepatitis B and HBV infection-related injury.

The IFNs are a family of proteins with an important role in protection against viral infections, tumor growth, inflammation and angiogenesis [17]. The IFNs are divided into three classes whereby the first two are most important from an immunological point of view. In humans, the main Type I IFNs consist of IFN- $\alpha$ (with 13 subtypes) and IFN- $\beta$ ( 1 subtype). IFN- $\alpha$ is produced in large amounts by plasmacytoid dendritic cells, whereas IFN- $\beta$ is mainly produced by fibroblasts.

IFN- $\alpha$ has been successfully used for the treatment of hepatitis [18] and we wondered if IFN- $\alpha$ has any effects on IL-17 and IL-10. In this study, we found that IFN- $\alpha$ 2a could inhibit IL-17 expression and increased IL-10 production by PBMCs from CAHB patients and controls. Further experiments using isolated $\mathrm{CD} 4^{+} \mathrm{T}$ cells from healthy controls showed similar results.

In our CAHB patients, we observed a stronger induction of IL-10 as compared with controls following incubation of PBMCs with IFN- $\alpha$. This result seems to suggest that a latent negative regulatory mechanism

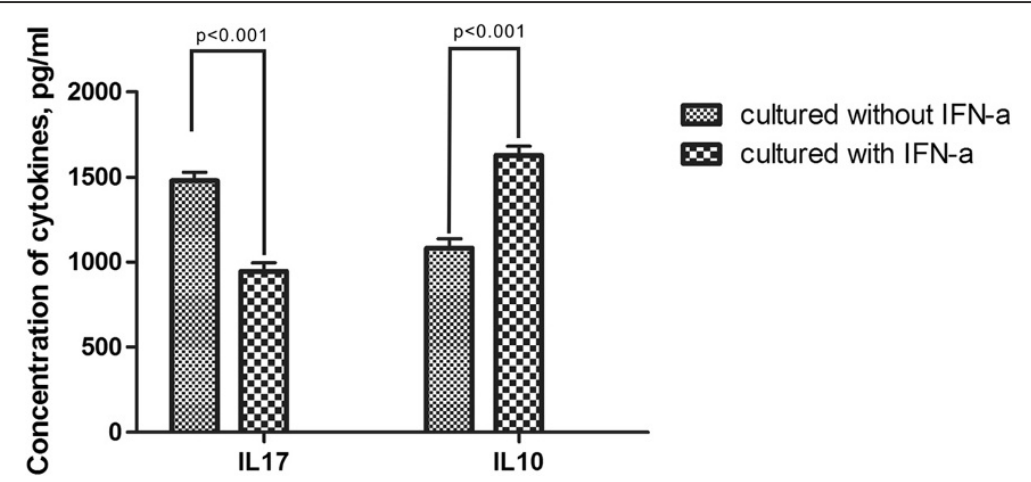

Figure 4 The Expression of IL-17 and IL-10 in by CD4+ T cells from healthy controls $(n=32)$ cultured with or without rhIFN- $a$ 2a. CD4+ $T$ cells from healthy controls $(n=32)$ were cultured with or without rhIFN-a $2 a$ at a concentration of $1 \times 10^{6}$ cells $/ \mathrm{ml}$ in combination with anti-CD3 $(5 \mathrm{mg} / \mathrm{ml})$ and anti-CD28 anti- bodies $(1 \mathrm{mg} / \mathrm{ml})$ for $72 \mathrm{~h}$. IL-10 production in the supernatant of cultured PBMCs was measured by ELISA. Columns represent meanvalues (mean + SEM). 


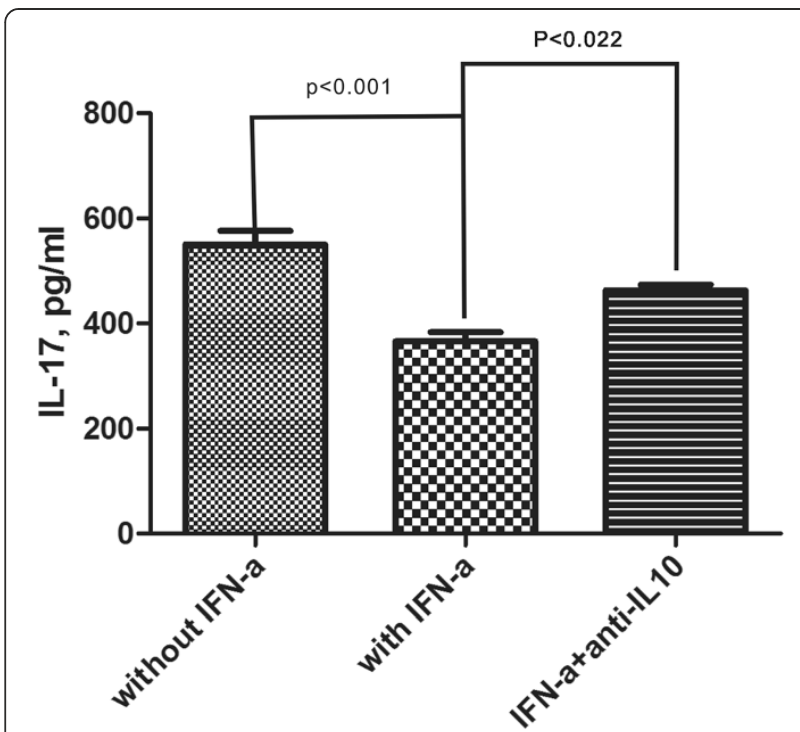

Figure 5 Anti-IL-10 antibody could partially neutralize the inhibitory effect of rhIFN-a 2a on IL-17 production by PBMCs from CAHB patients $(n=61)$. PBMCs from CAHB patients were cultured with or without rhIFN-a 2a, anti-IL-10 antibody in combination with anti-CD3 and anti-CD28 antibodies for $72 \mathrm{~h}$. Concentrations of IL-17 in the supernatants were detected by ELISA. Anti-IL-10 antibody $(10 \mathrm{mg} / \mathrm{ml})$ could partially neutralize inhibitory role of rhIFN-a 2a on IL-17 production. Columns represent mean values (mean + SEM).

already exists in these patients but that it is not triggered and is insufficient to suppress the overwhelming ongoing Th17 response. We also investigated whether IFN- $\alpha$ could exert its effects through up-regulation of IL-10. The results showed that IFN- $\alpha$ induced both PBMCs and $\mathrm{CD}^{+}{ }^{+} \mathrm{T}$ cells to produce IL-10. We then questioned whether IFN- $\alpha$ could inhibit IL-17 expression through an up-regulation of IL-10. Therefore, an experiment using an anti-IL-10 antibody was performed to examine underlying mechanisms of action of IFN- $\alpha$. Interestingly, we did find that the anti-IL-10 antibody blocked the inhibition of IL-17 production promoted by IFN- $\alpha$. These results suggest that the function of IFN- $\alpha$ is at least partially mediated by IL- 10 .

Also our results concerning the mechanism of action of IFN- $\alpha$ are consistent with those reported earlier in patients with other diseases. Zhang et al. [19] showed that IFN- $\alpha$ inhibited the differentiation of Th17 cells and production of IL-17 through up-regulating TLR7 expression in dendritic cells from patients with multiple sclerosis. It was reported that treatment with IFN- $\alpha$ could induce an increased frequency of $\mathrm{CD} 4^{+} \mathrm{CD} 25^{\text {high }} \mathrm{T}$ cells in PBMCs from MS patients [20]. However, Seya and coworkers [21] found that IFN- $\alpha$ from polyI:C-stimulated dendritic cells (DCs) inhibited the expansion of $\mathrm{CD}^{+} \mathrm{CD} 25^{+} \mathrm{FoxP} 3^{+}$ Tregs. Whether a down-regulatory effect of IFN- $\alpha$ on IL17 production by $\mathrm{T}$ cells, as observed in CAHB, is associated with an increased $\mathrm{CD}^{+} \mathrm{CD} 25^{+} \mathrm{FoxP}^{+}$Tregs needs further studies.

We performed these experiments using PBMCs from healthy control patients and ideally the data should also be confirmed using cells obtained from CAHB patients. Other cytokines such as IL-27 that are known to be induced by IFN- $\alpha$ and that can block the differentiation of Th precursor cells into Th17 cells may also be involved but were not included in our study $[22,23]$. Our experiments using isolated $\mathrm{CD}^{+}{ }^{+} \mathrm{T}$ cells from healthy controls showed levels of IL-17 and IL-10 in the culture supernatants much higher than in experiments with the same number of PBMCs. The absolute number of $\mathrm{CD}^{+} \mathrm{T}$ cells was much higher in such cultures and indicated that these cells are probably the main source of IL-17 and IL-10 in this in vitro model. On the other hand, $\delta \gamma$ $\mathrm{T}$ cells have also been shown to produce IL-17 and may play an important role. Further studies are needed to establish which T-cell subpopulations are subject to IFN- $\alpha$ regulation.

\section{Conclusions}

In conclusion, our study showed that IFN- $\alpha$ could down-regulate IL-17 levels in association with an upregulation of IL-10 in CAHB patients and healthy controls. The inhibitory effect of IFN- $\alpha$ on IL-17 production was partially mediated by IL-10. Further studies are needed to establish which T-cell subpopulations are subject to IFN- $\alpha$ treatment.

\section{Competing interests}

The authors declare that they have no competing interests.

\section{Authors' contributions}

All authors participated in the design, implementation, analysis and/or interpretation of the study. FC and JM led the clinical team at Department of Laboratory Medicine, and were involved in all phases of the study. PL and PC conducted all ELSA test. FC conducted FACS test and the statistical analysis. All the authors revised the manuscript critically for important intellectual content and approved the final version before submission.

\section{Acknowledgements}

This project was supported by The National Clinical Key Project (No.2010030). The authors thank all patients and healthy controllers in this study.

\section{Author details}

${ }^{1}$ Department of Laboratory Medicine, The First Affiliated Hospital of Chongqing Medical University, No. 1 Youyi Road, Chongqing, Yuzhong District, China. ${ }^{2}$ Department of Obstetrics and Gynecology, Assisited Reproductive Center, The First Affiliated Hospital of Chongqing Medical University, Chongqing 400016, China.

Received: 21 June 2013 Accepted: 30 January 2014 Published: 1 February 2014

\section{References}

1. Calvaruso V, Craxi A: Fibrosis in chronic viral hepatitis. Best Pract Res Clin Gastroenterol 2011, 25:219-230.

2. Di Bisceglie AM: Hepatitis B and hepatocellular carcinoma. Hepatology 2009, 49(5 Suppl.):S56-S60. 
3. Zhang JY, Zhang Z, Lin F, et al: Interleukin-17-producing CD4(+) T cells increase with severity of liver damage in patients with chronic hepatitis B. Hepatology 2010, 51:81-91.

4. Ye Y, Xie X, Yu J, et al: Involvement of Th17 and Th1 effector responses in patients with Hepatitis B. J Clin Immunol 2010, 30:546-555.

5. Hirohata S, Shibuya H, Tejima S: Suppressive influences of IFN-alpha on IL-17 expression in human CD4+ T cells. Clin Immunol 2010, 134:340-344.

6. Aman MJ, Tretter T, Eisenbeis I, et al: Interferon-alpha stimulates production of interleukin-10 in activated CD4 $+\mathrm{T}$ cells and monocytes. Blood 1996, 87:4731-4736.

7. de Waal MR, Haanen J, Spits H, et al: Interleukin 10 (IL-10) and viral IL-10 strongly reduce antigen-specific human $\mathrm{T}$ cell proliferation by diminishing the antigen-presenting capacity of monocytes via downregulation of class II major histocompatibility complex expression. J Exp Med 1991, 174:915-924.

8. Beissert S, Hosoi J, Grabbe S, Asahina A, Granstein RD: IL-10 inhibits tumor antigen presentation by epidermalantigen-presenting cells. J Immunol 1995, 154:1280-1286.

9. Fitzgerald DC, Zhang GX, El-Behi M, et al: Suppression of autoimmune inflammation of the central nervous system by interleukin 10 secreted by interleukin 27-stimulated T cells. Nat Immunol 2007, 8:1372-1379.

10. European Association for the Study of the Liver: EASL clinical practice guidelines: management of chronic hepatitis B. J Hepatol 2009, 50:227-242.

11. Asian-Pacific consensus statement on the management of chronic hepatitisB: a 2012 update. Hepatol Int 2012, 6:531-56.

12. Zhao $L$, Tang $Y$, You $Z$, et al: Interleukin-17 contributes to the pathogenesis of autoimmune hepatitis through inducing hepatic interleukin-6 expression. PLoS One 2011, 6:e18909.

13. Ge J, Wang K, Meng QH, et al: Implication of Th17 and Th1 cells in patients with chronic active hepatitis B. J Clin Immunol 2010, 30:60-67.

14. Wu W, Li J, Chen F, et al: Circulating Th17 cells frequency is associated with the disease progression in $\mathrm{HBV}$ infected patients. $J$ Gastroenterol Hepatol 2010, 25:750-757.

15. Li J, Wu W, Peng G, et al: HBcAg induces interleukin-10 production, inhibiting HBCAg-specific Th17 responses in chronic hepatitis B patients. Immunol Cell Biol 2010, 88:834-841.

16. Korn T, Bettelli E, Oukka M, Kuchroo VK: IL-17 and Th17 Cells. Annu Rev Immunol 2009, 27:485-517.

17. Meyer $\mathrm{O}$, et al: Interferons and autoimmune disorders. Joint Bone Spine 2009, 76:464-473.

18. Finter NB, Chapman S, Dowd P, et al: The use of interferon-alpha in virus infections. Drugs 1991, 42:749-765.

19. Zhang X, Jin J, Tang Y, Speer D, Sujkowska D, Markovic-Plese S: IFN-beta1a inhibits the secretion of Th17-polarizing cytokines in human dendritic cells via TLR7 up-regulation. J Immunol 2009, 182:3928-3936.

20. Penton-Rol G, Cervantes-Llanos M, Cabrera-Gomez JA, et al: Treatment with type I interferons induces a regulatory T cell subset in peripheral blood mononuclear cells frommultiple sclerosis patients. Int Immunopharmacol 2008, 8:881-886.

21. Kubota N, Ebihara T, Matsumoto M, et al: IL-6 and IFN-alpha from dsRNAstimulated dendritic cells control expansion of regulatory T cells. Biochem Biophys Res Commun 2010, 391:1421-1426.

22. Pirhonen J, Siren J, Julkunen I, Matikainen S: IFN-alpha regulates Toll-like receptor-mediated IL-27 gene expression in human macrophages. J Leukoc Biol 2007, 82:1185-1192.

23. Diveu C, McGeachy MJ, Boniface K, et al: IL-27 blocks RORc expression to inhibit lineage commitment of Th17 cells. J Immunol 2009, 182:5748-5756.

doi:10.1186/1471-2334-14-55

Cite this article as: Cui et al.: IFN- alpha blocks IL-17 production by peripheral blood mononuclear cells in patients with chronic active hepatitis B Infection. BMC Infectious Diseases 2014 14:55.

\section{Submit your next manuscript to BioMed Central and take full advantage of:}

- Convenient online submission

- Thorough peer review

- No space constraints or color figure charges

- Immediate publication on acceptance

- Inclusion in PubMed, CAS, Scopus and Google Scholar

- Research which is freely available for redistribution

Submit your manuscript at www.biomedcentral.com/submit 